\title{
Performance Analysis of the Deep Learning Method for Medical Cases
}

\author{
Dian Pratiwi \\ Trisakti University \\ Faculty of Industrial Technology, \\ Department of Informatics \\ Engineering \\ Jakarta, Indonesia
}

\author{
Anung B. Ariwibowo \\ Trisakti University \\ Faculty of Industrial Technology, \\ Department of Informatics \\ Engineering \\ Jakarta, Indonesia
}

\author{
Dimmas Mulya \\ Trisakti University \\ Faculty of Industrial Technology, \\ Department of Informatics \\ Engineering \\ Jakarta, Indonesia
}

\begin{abstract}
The development of technology is moving very rapidly, especially in the medical field. This is due to the increasing number of cases of disease requiring the diagnostic ability of a Medical Expert to analyze, but not all Medical Personnel can carry out an in-depth analysis with a Medical Expert. Therefore, a lot of research has been carried out to make technology to help in medical process. Having research that can mimic a medical expert's ability to diagnose disease can increase the chances of a patient being saved before it's too late. There are many methods used in research to assist in the process of diagnosing a patient's disease. Deep learning method is the one that is used. Deep Learning method is split up into several derived methods, that methods are Deep Neural Networks, Convolutional Neural Networks, and Recurrent Neural Networks. With analyzing abilities each derived methods, it can make it easier for further research to create or develop better research than before in order to advance technology in the medical field...
\end{abstract}

\section{Keywords}

Artificial Intelligence, Deep Learning, Disease Diagnosis, Deep Neural Network, Convolutional Neural Network, Recurrent Neural Network.

\section{INTRODUCTION}

Technology advances in the Medical Sector move very quickly along with the times. With this rapid progress, many medical personnel are assisted in taking actions and decisions quickly to treat patients and can reduce the possibility of Human Error occurring in the diagnosis process. This can be done because with this technology Medical Personnel can take fast steps without the direct supervision of Medical Experts, thus increasing the possibility for patients to survive the illness they are suffering from. The development of this technology can be in the form of decision support or decision making based on artificial intelligence which is used in the diagnostic process of Medical Personnel.

There are many methods used in technological developments in the Medical Field, such as Expert Systems and Deep Learning. Deep Learning is one of the methods of the branch of artificial intelligence which can study patterns from a set of data so that it can provide predictions to users based on the patterns they are learning. [1] The deep learning method is often used in the development of medical technology due to its ability to study data patterns, so that the higher the hours of use, the higher the accuracy in providing predictions or decisions to medical personnel. [2] Therefore, many studies use deep learning methods to be able to make quick decisions without direct medical expert supervision.

By carrying out this research, the researcher hopes to provide information and insights regarding the capabilities of the deep learning method in advancing the medical field. So that it can provide the right direction to develop Deep Learning for the Medical Field and can save many patient lives before it's too late.

\section{LITERATURE REVIEW}

\subsection{Artificial Neural Network}

Artificial Neural Network (ANN) is one of the methods of Artificial Neural Network approach which imitate the work of human's brain. That approach then implement into a source code to solve a problem. There are three elements of Artificial Neural Network, namely weight, threshold, and activation function. $[3,4]$

Artificial Neural Networks have three kind of variety [5] :

a) Single Layer is the simplest form of Artificial Neural Network.This variety called as that because this variety doesn't have Hidden Layer. The way this variety works is by projecting input value on Input Layer to the Output Layer. Fig. 1 show the example of Single Layer.

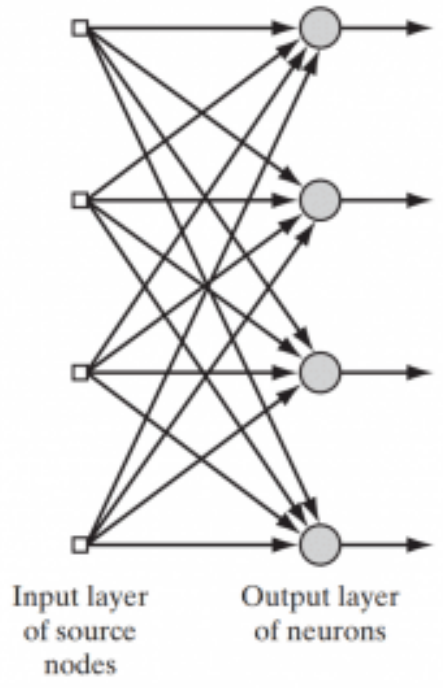

Fig 1: Single Layer ANN [5]

b) Multi Layer is a form of Single Layer ANN with Hidden 
Layer. Hidden Layer itself is a functions that process further the input value from the Input Layer so that it can provide an appropriate output value on the Output Layer. The way it works is similar to the Single Layer but the input value from the Input Layer is projected onto the Hidden Layer first before going to the Output Layer. Fig. 2 show the example of a Multi Layer ANN.

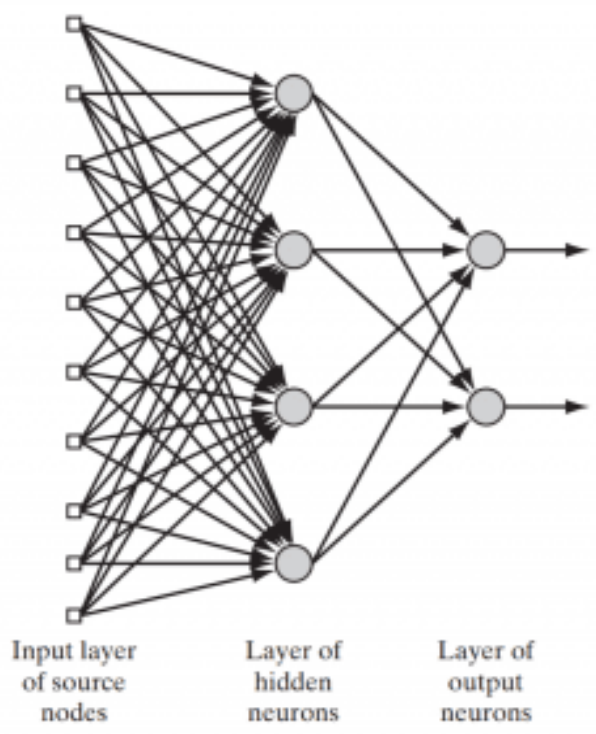

Fig 2: Multi Layer ANN [5]

c) Reccurent Network is a variety of ANN with form of a loop or giving input to itself. So that the input results will be reused as the input value after process. Fig. 3 show the example of a Recurrent Network type.

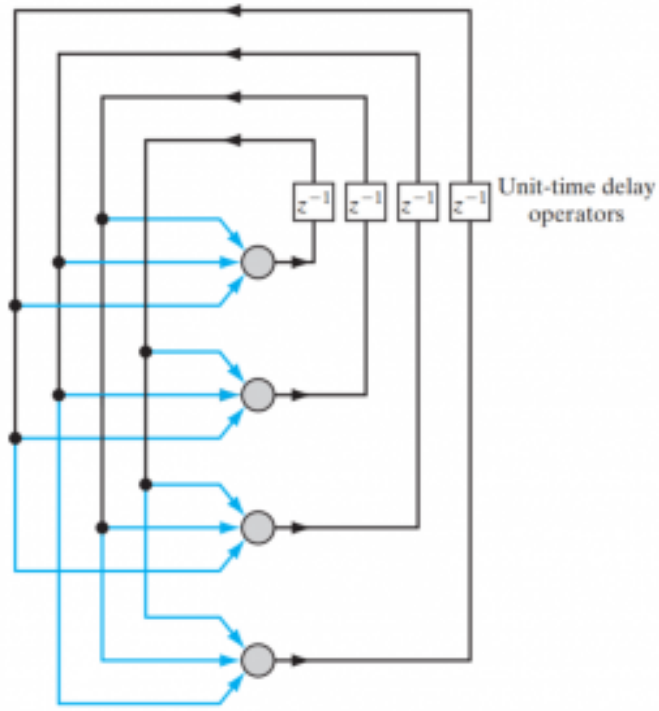

Fig 3: Recurrent Network ANN [5]

\subsection{Deep Learning}

Deep Learning is a form of Machine Learning where there is a learning process from experience, so that it can allow computers to understand patterns of a data set. This can happen because of the concept of hierarchy that allows computers to learn complex concepts by building them from more simple. [2] The description that concept is referring the Hidden Layer.

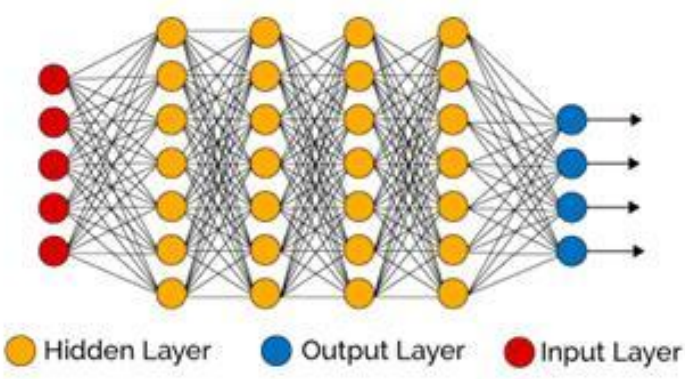

Fig 4: Deep Neural Network

Deep Learning itself is related to ANN. It is because the concept of Deep Learning itself is a learning process from Deep Neural Networks, Convolutional Neural Networks, and Recurrent Neural Networks. [6,7]

a) Deep Neural Network is one of the methods of deep learning. The Deep Neural Network itself is formed from an Artificial Neural Network which has more or the same two hidden layers. Hence, a Deep Neural Network is some of the time alluded to as a Multi Layer Perceptron. In common, Deep Neural Networks are utilized to memorize and learn the patterns so that they can be implemented more broadly. One way to train from a Deep Neural Network is the Back-Propagation method. The use of the formula in the Deep Neural Network only need differential process for each Hidden Layers contained in the Deep Neural Network. [2,6] Overall, Deep Neural Networks are supperior than wider Neural Networks with less layers. This is because Deep Neural Networks have more layers than Artificial Neural Networks so that there will be a lot of transformation processes. The transformation in question is the process of changing the input value into a representative value for the Hidden Layer. Then, another advantage of a Deep Neural Network is that it has the ability to convert non-linearly seperable data to linearly separable by the transformation process through the Hidden Layer. In addition, Deep Neural Networks can also look for non-linear Decision Boundries, so that they can draw conclusions on nonlinear interactions between features. [6] Here's an example of a deep neural network in Fig. 5.

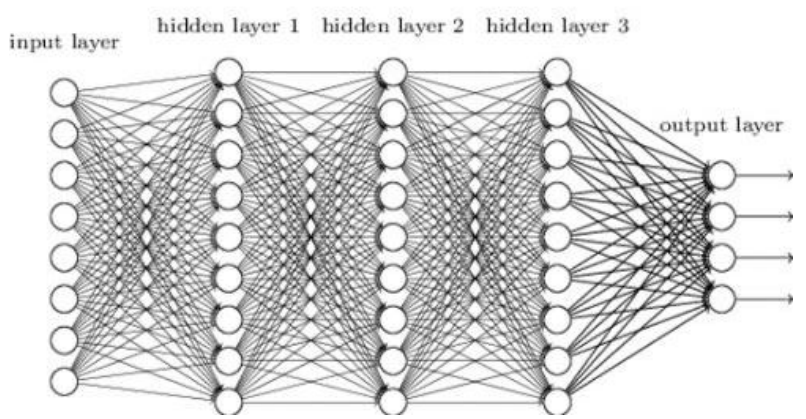

Fig 5: Example of Deep Neural Network

b) Convolutional Neural Network is taken from mathematics concepts, where the calculation points to the main estimation of two covering function. Convolutional Neural Network has the main function of recognizing an image as a dimensional object. In general, the colors in digital images can be assembled into the red-green-blue (RGB) color coding for humans to see the color spectrum. From that spectrum, the Convolutional Neural Network can take as input from an pictureencompassed by a square box. Fig. 6 show the architecture of a Convolutional 


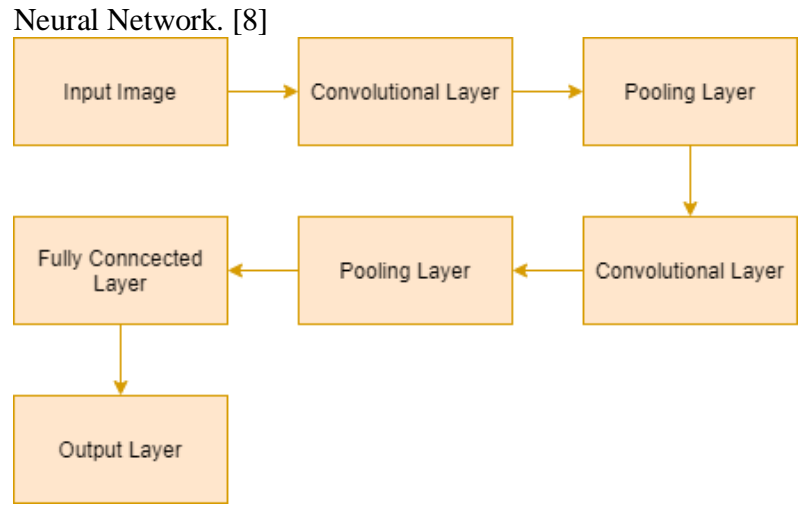

Fig 6. Architecture of Convolutional Neural Network

c) Recurrent Neural Network is the concept of a Neural Network to solve problems in the Neural Network process, the network is required to have a memory of dynamic output and input due to the random and biased initialization of weights. Therefore this method is called Reccurent because it has a special feature that is, it does similar work with each element sequentially. $[2,8,9]$

\subsection{Deep Learning Research in Medical Field}

Deep Learning has been widely implemented in the Medical Sector, both in providing support for diagnostic decisions and making decisions for diagnosis. In general, the deep learning process is carried out through 2 stages, namely learning and testing. The Learning Stage is the stage of training Deep Learning as a basis for knowledge. Then enter the Trial Phase to test the accuracy and accuracy of the Deep Learning capabilities.

Data in the Medical Sector has quite a variety of forms, so it requires a method that is in accordance with the medical data to be used, so that the data use process can run well. In addition, an adequate amount of data is needed for the Learning Stage in order to produce good output accuracy.

The usage of the Deep Neural Network method is carried out in cases that use a general dataset, taken in a certain time, and are not continuous (e.g. blood test dataset). Each value of each variable per case will be used as a benchmark in the Learning and Trial Phase of the Deep Neural Network. There are many studies using this method to indentify many different diseases with previous medical data, namely as follows :

a) K. H. Miao et al. conducted research to detect coronary heart using the Deep Neural Network method. This Deep Neural Network can study variable patterns for people with coronary heart disease. The variables in question are medical data from patients such as age, sex, cholesterol level and others. [10]

b) A. Caliskan et al. conduct research to detect Parkinson's disease using deep learning. The data used is voice measurement from the patient. Data voice measurement is used because the early symptoms of Parkinson's can be detected by a patient's speech impediment. The deep learning method is used to study patterns of speech impediments of a patient so that they can detect parkisone early on. [11]

c) Z. Yao et al. conduct research to indentify liver disease based on blood test results. The deep learning method is utilized to study the pattern of anomalies in the blood test results of people with liver disease. [12] d) J. Geraci et al. conducted a study to detect depression in young children using the Deep Neural Network method based on the medical history (EMR) of adolescents. [13]

e) D. Pratiwi et al. conducted a study using the DNN method to indentify the severity of dengue fever. With data from the patient's blood test results, the deep learning method was used to study the pattern of each degree of dengue fever severity. [14,15]

Then, the usage of the Convolutional Neural Network method is done in cases that use a dataset in the form of a set of images (e.g. X-Ray scan results). Convolutional Neural Network can study two dimensional color pixel data patterns that have been processed through image processing methods first. There are many studies used CNN method to indentify many diseases using previous medical data, namely as follows

a) S. Pereira et al., G. Urban er al., A. Devy et al., and D. Zikic et al. conducted a study to diagnose brain tumors using the Convolutional Neural Network method. The data used are images of MRI scans. With the Convolutional Neural Network, the image pattern of the brain tumor on the MRI scan image can be determined. [16-19]

b) C. Yao et al. conducted research to detect gallstones based on their chemical composition. The data used are the results of tomography and resonance imaging of gallstones. So that by studying patterns from these data, the Convolutional Neural Network can provide output in the form of gallstones suffered by patients. [20]

c) D. $\mathrm{Lu}$ et al. conduct research to detect Alzheimer's disease. With the Alzheimer's Disease Neuroimaging Initiative (ADNI) dataset from site http://adni.loni.usc.edu/ in the form of MRI and FGDPET images, researchers can show that the Convolutional Neural Network can study patterns from these datasets and provide the appropriate output. [21]

d) H. Wang et al. conducted a study to diagnose diseases of the thoracic bone with the Convolutional Neural Network method. With the dataset of chest X-rays, Convoutional Neural Network can study the pattern of $\mathrm{X}$-rays to diagnose thoracic bone disease. [22]

e) A. Esteva et al. conducted a study to detect skin cancer using the Convolutional Neural Network method. The dataset used is a photo of skin images that have anomalies or symptoms of cancer. [23]

f) D. Pratiwi et al. conduct research to detect the severity of osteoporosis. The dataset used is an image of bone X-ray scna results from bones with varying degrees of osteoporosis severity. [24]

Then, the usage of the Recurrent Neural Network method is used in cases where the dataset has continuity and is continuity between one another (e.g: EKG dataset). The process of this method is different from other Neural Networks because it uses a looping output value that is used as a return input. So it is suitable to be used to study mutually sustainable data patterns. There are several studies using this method to detect various diseases using previous medical data, namely as follows :

a) R. Khoirani conducted a study to detect atrial fibrillation heart disease based on signals from the ECG results. The Recurrent Neural Network method can study patterns of 
abnormal beat rhythms such as fast and irregular so that it can provide output to detect atrial fibrillation heart disease. [25]

b) A. A. Petrosian et al. conducted research to detect early symptoms of Alzheimer's using the Recurrent Neural Network method. The data used are EEG signals. By studying the abnormal EEG signal, this method can detect the early symptoms of Alzheimer's. [26]

c) E. Choi et al. conducted a study to detect heart failure based on a pattern from the EHR dataset using the Recurrent Neural Network method. [27]

d) S. B. Babu et al. conducted a study to foresee different types of disease based on medical history data provided by the UCI with various variables. [28]

\section{PROCEDURE}

In general, each research using the same method will go through the same process as well. This is because the concept of deep learning methods uses the weight change process in the Neural Network. However, in order to adapt to this process, it is necessary to process the dataset first. Then there is also an additional process so that the results of the Deep Learning process provide higher accuracy.

\subsection{Deep Neural Network}

In the research of K. H. Miao et al. To detect coronary heart disease, the dataset used contained 28 variables as characteristics of those who suffer from coronary heart disease and are healthy. Of the 28 variables, the system will study the pattern of these characteristics by epoching 5000 times and using a learning rate of 0.00005 . The similarity in the process of the research is also the same in Z's study. Yao et al. to detect liver disease. However, from other studies such as research by J. Geraci et al. to detect depression in youth using a more complex dataset requires additional methods in processing the dataset used. In the research of J. Geraci et al. adding the Brute Force Search method in getting the desired data, because the dataset used is in the form of unstructured writing. In addition to adding methods to processing the dataset, there are also adding process stages to the Deep Neural Network method in order to process the dataset better. In Research A. Caliskan et al. to detect Alzheimer's disease by adding an autoencoder and softmax classifier to the process of the Deep Neural Network. This was also carried out by the research of D. Pratiwi et al., Namely adding the Min-Max method and the Dropout Mechanism to prevent overfitting.

\subsection{Convolutional Neural Network}

In studies done by A. Davy et al., G. Urban et al., S. Pereira et al., And D. Zikic et al. using the same method, namely, Convolutional Neural Network but using variant procedures. In the research of S. Pereira et al. using a $3 \times 3$ filter process for the framework model.In the study of G. Urban et al. using the 3D convolutiobnal filter procedure to process the 3D-CNN architecture. Then in A. Davy's research, he used the two-way CNN procedure to process the image completely and partially. Then in the research of D. Zikic et al. using the 3D input procedure to $2 \mathrm{D}$. The method they use aims to process the data they use so that it can provide the best output.

$\mathrm{n}$ the study by C. Yao et al. used a simple process from the Convolutional Neural Network to study the pattern of gallstone resonance imaging results. By being able to study the pattern of resonance of each type of gallstone and be able to give a classification. Same as in the research of D. Lu et al. in diagnosing Alzheimer's disease by doing image preprocessing first and research D. Pratiwi to detect osteoporosis from the results of X-Ray images.

In the research of $\mathrm{H}$. Wang et al. the same also uses a simple process from the Convolutional Neural Network but there is an additional method for processing the dataset before use, namely changing the X-Ray image resolution to $224 \times 224$, which is then carried out by epoch as much as 50000 with a learning reate of 0.001 .

\subsection{Recurrent Neural Network}

In R. Khoirani's research, he used the Recurrent Neural Network to detect atrial fibrillation heart disease based on the signal from the EKG. The process is carried out as in general by training the Recurrent Neural Network so that it can produce accurate output. This is also done in the research of A. A. Petrosian et al. which uses the EEG dataset for early diagnosis of Alzheimer's disease and E. Choi's research uses HER data to detect heart failure.

Research conducted by S. B. Babu et al. using the same method but there are additional procedures that are included in the Recurrent Neural Network process, namely Gray Wolf Optimization and RNN-based Auto Encoder.

\section{RESULT AND DISCUSSION}

From each of the studies that have been described, it can be concluded that the methods of deep learning produce high accuracy to provide decisions or decision support. This is due to the ability of Deep Leraning itself because it can improve the weight of each usage calculation so that the higher the hours of use, the better the output that Deep Learning can provide. Therefore, each of the above studies states that deep learning has better accuracy than other methods.

For the Deep Neural Network method, as in the research conducted by J. Geraci et al. managed to achieve an accuracy of $77 \%$ and a specificity of $68 \%$. Then in the research of D. Pratiwi et al. also managed to achieve an accuracy rate of $87.5 \%$. Furthermore, in study done by Z. Yao et al. successful in proving that the ability of the Deep Neural Network has a higher accuracy than Random Forest and Logistic Regression methods then in A.Caliskan's study succeeded in showing that the accuracy of the Deep Neural Network is higher than the Naïve Bayes and Decision Tree methods.

Then in the Convolutional Neural Network method, research conducted by S. Pereira managed to achieve an accuracy rate of $88 \%$. Furthermore, in research conducted by $\mathrm{H}$. Wang et al. managed to achieve an accuracy rate of $78.1 \%$. Then in research a. Esteva et al. managed to achieve an accuracy of $93.33 \%$. Then in D. Pratiwi's research managed to achieve an accuracy rate of $66.6 \%$.

In the Recurrent Neural Network method used by E. Choi et al. to detect heart failure, it has achieved an accuracy rate of $77.11 \%$ and can be proven to be better than Logistic Regression method, Multilayer Perceptron, Support Vector Machine method, and K-Nearest Neighbors. Then in S. Babu's research also succeeded in showing an accuracy rate of 98.23\% and it can also be proven that it is also better than the Fuzzy Particle Swarm Optimization, Fuzzy Neural Network, Decision Tree, Bagging Algorithm, and Backpropagation Neural Network methods.

Based on explanation above, it can be concluded that the capabilities of deep learning methods are more accurate than the capabilities of other prediction methods. This is because the system in Deep Learning is not static, but there will be learning back along with use, so that the system will be able 
to produce better output than before.

\section{CONCLUSION}

Various studies have been successfully carried out to support the medical field in providing diagnosis or assisting in the form of providing decision support to Medical Personnel. From each of these studies, it can prove that the ability of the deep learning method itself. In addition to providing a high level of accuracy, the Deep Learning method has been proven to be better than other prediction methods. This is due to the ability of deep learning methods to develop on their own with the number of uses. The implementation of deep learning is based on the type of dataset that you want to use as a research target. By processing the right data, selecting the appropriate method and adding the necessary methods, it can increase the accuracy of deep learning itself. This has been proven successfully by research from each of the Deep Learning methods with a fairly large accuracy, 83.67.\%, Deep Neural Network method, 93.33\%. Convolutional Neural Network method 98.23\%.Recurrent Neural Network method.

\section{ACKNOWLEDMENT}

This research is fully funded by Trisakti and is intended to commemorate the late Sri Mulyani for all her support and affection for the author

\section{REFERENCES}

[1] S. Chae, S. Kwon, D. Lee, "Predicting Infectious Disease Using Deep Learning and Big Data," International Journal of Environmental Research and Public Health, Vol. 15, No. 8, Jul. 2018.

[2] I. Goodfellow, Y. Bengio, A. Courville, Deep Learning. Cambridge: MIT Press, 2016.

[3] A. S. Kurniawansyah, "ImplementasiMetode Artificial Neural

Network DalamMemprediksiHasilUjianKompetensiKebidanan,” JurnalPseudocode, Vol. 5, No. 1, Feb. 2018.

[4] Y. Setiowati, Class Lecture. Topic: "JaringanSyarafTiruan (N eural Network)," TeknikInformatika, PoliteknikElektronikaNegeri Surabaya, Surabaya, JawaTimur, Sep. 4, 2014.

[5] A. Yanuar, "Artificial Neural Network (ANN)," ugm.ac.id, May. 28, 2018.[online]. Tersedia: https://machinelearning.mipa.ugm.ac.id/2018/05/24/artifi cial-neural-network-ann/. [Access Oct. 10, 2020].

[6] J. W. G. PengenalanKonsepPembelajaranMesindan Deep Learning. 1.4 Ed., Tokyo: Research Gate, 2020, [ebook]. $\quad$ Tersedia: https://www.researchgate.net/publication/323700644_Pe ngenalan_Pembelajaran_Mesin_dan_Deep_Learning [Oct 12, 2020].

[7] Y. LeCun and M. A. Ranzato, "Deep Learning Tutorial" presented at IMCL, Atlanta, US, 2013.

[8] T. Poongodi, D. Sumanthi, P. Suresh, B. Balusamy, "Deep Learning Techniques for Electronic Health Record (EHR) Analysis," Bi-inspired Neurocomputing, Vol. 903, pp. 73-101, July. 2020.

[9] K. Gregor, I. Danihelka, A. Graves, D. J. Rezende, D. Wierstra, "DRAW: A Recurrent Neural Network For Image Generation," arXiv preprint arXiv: 1502.04623. Feb. 16, 2015.
[10] K. H. Miao, J. H. Miao, "Coronary Heart Disease Diagnosis using Deep Neural Networks," International Journal of Advanced Computer Science and Applications, Vol. 9, No. 10, pp. 1-8, 2018.

[11] A. Caliskan, H. Badem, A. Basturk, et al., "Diagnosis Of The Parkinson Disease By Using Deep Neural Network Classifier," IU-JEEE, Vol. 17, No. 2, pp. 3311-3318, 2017

[12] Z. Yao, J. Lia, Z. Guan, Y. Ye, Y.Chen, "Liver disease screening based on densely connected deep neural networks," Neural Networks, Vol. 123, pp. 299-304, March. 2020.

[13] J.Geraci, P. Wilansky, V. D. Luca, et al., “Applying deep neural networks to unstructured text notes in electronic medical records for phenotyping youth depression", BMJ Journal Evidence-Based Mental Health, Vol. 20, pp. 8387, 2017.

[14] D. Pratiwi, A. B. Ariwibowo, "Dengue Haemorrhagic Fever (DHF) Severity Detection by Using Neural Network Technique based on Human Blood Components," International Journal of Mechanical \& Mechatronics Engineering, Vol. 17, No. 3, June. 2017

[15] D. Pratiwi, G. B. Santoso, L. Muslimah, et al., "An Intelligent Dengue Hemorrhagic Fever Severity Level Detection Based On Deep Neural Network Approach," JurnalllmuKomputerdanInformasi, Vol. 12, No. 2, pp.57-66, 2019.

[16] S. Pereira, A. Pinto, V. Alves and C. A. Silva, "Brain Tumor Segmentation Using Convolutional Neural Networks in MRI Images," IEEE Transactions On Medical Imaging, Vol. 35, No. 5, pp. 1240-1250, May. 2016.

[17] A. K. Bhoi, P. K. Mallick, C. M. Liu, "Multi-modal Brain Tumor Image Segmentation Using Deep Convolutional Neural Networks," in Bio-inspired Neurocomputing Vol. 903, V. E. Balas, Singapore: Springer, 2020. [e-book] Available https://doi.org/10.1007/978-981-15-5495-7.

[18] A. K. Bhoi, P. K. Mallick, C. M. Liu, "Brain Tumor Segmentation with Deep Neural Networks," in Bioinspired Neurocomputing Vol. 903, V. E. Balas, Singapore: Springer, 2020. [e-book] Available : https://doi.org/10.1007/978-981-15-5495-7.

[19] A. K. Bhoi, P. K. Mallick, C. M. Liu, "Segmentation of Brain Tumor Tissues with Convolutional Neural Networks," in Bio-inspired Neurocomputing Vol. 903, V. E. Balas, Singapore: Springer, 2020. [e-book] Available : https://doi.org/10.1007/978-981-15-5495-7.

[20] C. Yao, S. Wu, Z. Liu et al., "A deep learning model for predicting chemical composition of gallstones with big data in medical Internet of Things," Future Generation Computer Systems, Vol. 94, pp. 140-147, May. 2019.

[21] D. Lu, K. Popuri, G. W. Ding, et al., "Multimodal and Multiscale Deep Neural Networks for the Early Diagnosis of Alzheimer's Disease using structural MR and FDG-PET images," Scientific Reports, Vol. 8, No. 1, pp. 1-13, 2018.

[22] H. Wang, Y. Xia, "ChestNet: A Deep Neural Network for Classification of Thoracic Diseases on Chest Radiography," arXiv preprint arXiv: 1807.03058, July. 9, 
2018.

[23] A. Esteva, B. Kuprel, R. A. Novoa, "Dermatologist-level classification of skin cancer with deep neural networks," Nature, Vol. 542, No. 7639, pp. 115-118, 2017.

[24] D. Pratiwi, D. D. Santika, B. Pardamean, "An Application Of Backpropagation Artificial Neural Network Method for Measuring The Severity of Osteoarthritis," International Journal of Engineering \& Technology, Vol. 11, No. 3, June. 2011.

[25] R. Khoirani, S. Nurmaini, "KlasifikasiPenyakitJantung Atrial Fibrillation (AF) DenganMenggunakanMetode Recurrent Neural Network (RNN) PadaKasus Multiclass," Doctoral Dissertation, Sriwijaya University, 2019.
[26] A.A. Petrosian, D.V. Prokhorov, W. Lajara-Nanson, et al., "Recurrent neural network-based approach for early recognition of Alzheimer's disease in EEG," Clinical Neurophysiology, Vol. 112, pp. 1378-1387, 2001.

[27] E. Choi, A. Schuetz, W. F. Stewart, et al., "Using recurrent neural network models for early detection of heart failure onset," Journal of the American Medical Informatics Association, Vol. 24, No. 2, pp. 361-370, 2017.

[28] B. S. Babu, A. Suneetha, G. C. Babu, "Medical disease prediction using Grey Wolf optimization and auto encoder based recurrent neural network" Periodicals of Engineering and Natural Sciences, Vol. 6, No. 1, pp. 229-240, June. 2018. 